\title{
Symmetry Compensation using a H-Bridge Multilevel STATCOM with Zero Sequence Injection
}

\author{
R.E. Betz ${ }^{*}$, T. Summers ${ }^{\dagger}$, T. Furney ${ }^{\ddagger}$ \\ School of Electrical Engineering and Computer Science \\ University of Newcastle, NSW, Australia, 2308 \\ Email: *Robert.Betz@newcastle.edu.au, †Terry.Summers@newcastle.edu.au, \\ tTodd.Furney@studentmail.newcastle.edu.au
}

\begin{abstract}
This paper examines the use of a H-bridge cascade multilevel STATCOM for symmetry compensation. One of the particular problems H-bridge based STATCOMs have when used in these applications is maintaining correct voltages on the H-bridge capacitors for each of the individual phases of the STATCOM. This difficulty is the result of average real power flowing in or out of the individual phase legs. A solution has already been published for the delta connected STATCOM, but has not, until this paper, been solved for the wye connected topology. This paper uses a new approach to solve the phase leg power balance problem using zero sequence current and voltage injection. It allows the individual phase voltages for the both the delta and wye connected cascaded H-bridge STATCOM to be controlled. Furthermore, when implemented for the delta connected STATCOM it leads to a more elegant and parameter independent control system architecture.
\end{abstract}

\section{INTRODUCTION}

Unbalanced voltages in a power system can result from a number of causes, some of which are: lack of symmetry in transmission line impedances; large single phase loads; faulty power factor correction capacitor banks; open delta or wye transformers; a fuse blows in a single phase resulting in "single phasing"; and different single phase loads on each phase in a three phase system.

Unbalance has a particularly bad effect in three phase equipment such as electrical machines. These effects have been documented in a number of places $[1,2]$ and have also been included in standards for electrical machines (e.g. National Electrical Machines Association (NEMA) standards in the USA). Table I shows how the lifetime and efficiency of an induction machine varies with unbalance. The lifetime of the machine is mainly effected through the degradation of the insulation of the machine due to increased temperature of operation. In addition to the reduction in lifetime, unbalance also affects the efficiency of the machine and can largely nullify the benefits of the use of high efficiency machines. A more subtle effect is the loss of production due to lower power being available and higher slips in the machine.

Remark 1: A rule-of-thumb for voltage unbalance and the resultant percentage temperature rise above the rated temperature is: $\%$ temperature increase $=(\% \text { voltage unbalance })^{2} \times 2$. Therefore a $3 \%$ voltage unbalance will result in the winding temperature rising by $3^{2} \times 2=18 \%$. Under these conditions the lifetime of the machine is reduced from 20 years (with no unbalance) to 2 years! [1]
Remark 2: In addition to the effects on induction machines, imbalance can also have effects on Adjustable Speed Drive (ASD) rectifier front ends, resulting in an increase in the imbalance in the system and in some cases resulting in tripping from excessive currents in single phases[3].

Cascade H-bridge multilevel converter topologies have been receiving considerable attention recently $[4,5]$. The reasons are: H-bridge based multilevel converters are modular; it is feasible to have a large number of levels allowing direct grid connection (without an interposing transformer); the number of components required in the converter increases linearly with the number of levels; and whilst there are voltage balance problems with the converter they are not as difficult as those for the neutral point clamped or flying capacitor converter with large level numbers. With neutral point and flying capacitor topologies the number of components increases as a quadratic with respect to the number of levels. The basic structure for the single leg of a 5 level H-bridge multilevel converter is shown in Figure 1.

The main disadvantage of the H-bridge multilevel cascade converter is it is not suitable for handling real power. Each H-bridge has to be fed from an isolated power supply, and therefore large numbers of isolated power supplies are required to handle the real power flow for high level number systems. However, if one only has to deal with reactive power, then

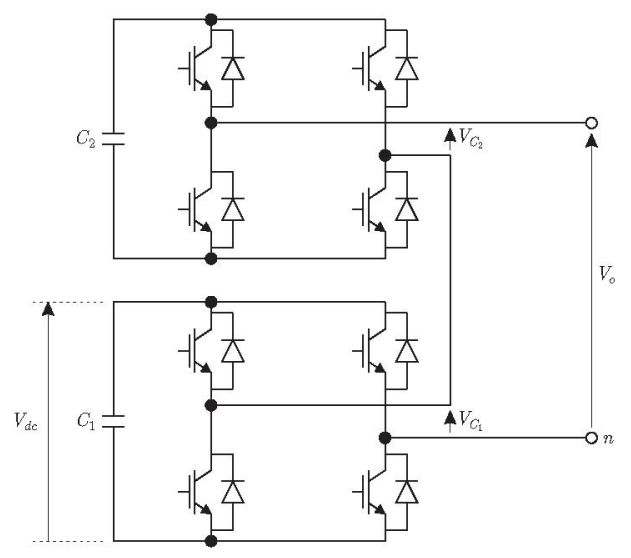

Fig. 1. Five level H-bridge multilevel cascade converter leg. 
TABLE I

EFFECTS OF UNBALANCE ON TEMPERATURE, LOSSES, EFFICIENCY AND LIFE TIME OF AN INDUCTION MACHINE AT RATED LOAD [1].

\begin{tabular}{c|c|c|c|c}
\hline $\begin{array}{c}\text { \% voltage } \\
\text { unbalance }\end{array}$ & $\begin{array}{c}\text { Winding temp } \\
(\mathrm{C})\end{array}$ & $\begin{array}{c}\mathbf{I}^{2} \mathbf{R} \text { losses - \% } \\
\text { of total losses }\end{array}$ & $\begin{array}{c}\text { Efficiency } \\
\text { reduction }\end{array}$ & $\begin{array}{c}\text { Expected } \\
\text { winding life } \\
\text { (yrs) }\end{array}$ \\
\hline \hline 0 & 120 & $30 \%$ & none & 20 \\
1 & 130 & $33 \%$ & Up to $0.5 \%$ & 10 \\
2 & 140 & $35 \%$ & $1 \rightarrow 2 \%$ & 5 \\
3 & 150 & $38 \%$ & $2 \rightarrow 3 \%$ & 2.5 \\
4 & 160 & $40 \%$ & $3 \rightarrow 4 \%$ & 1.25 \\
5 & 180 & $45 \%$ & $\geq 5 \%$ & $<1$ \\
\hline
\end{tabular}

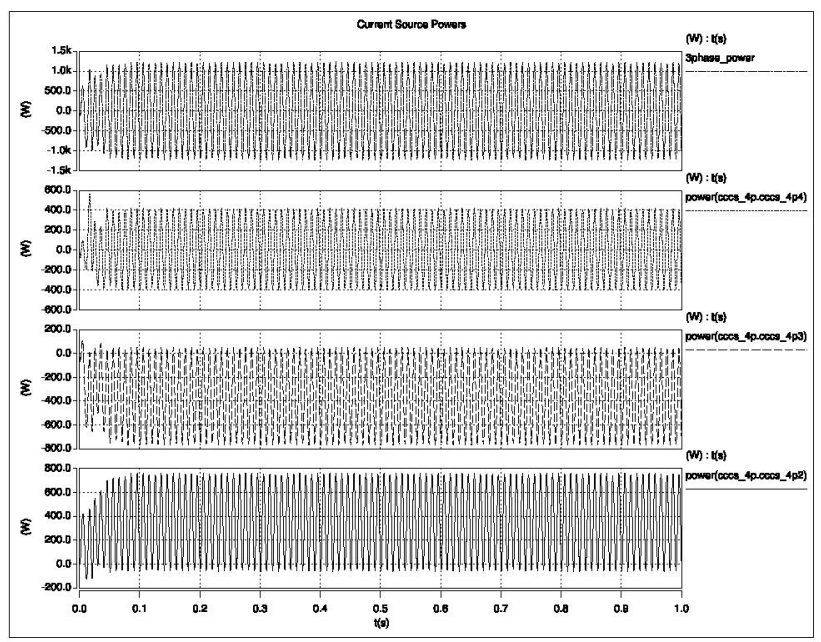

Fig. 2. Instantaneous power in a wye connected STATCOM legs under a open circuit phase unbalance condition.

only capacitors are required for each H-bridge in the cascade. ${ }^{1}$ Therefore the H-bridge topology is very suitable for reactive power compensation and active filtering in power systems.

H-bridge STATCOMs are usually arranged as a wye connected structure so that each phase leg of the STATCOM can be rated for the phase voltage and not the line-to-line voltage (as would be the case for a delta connected STATCOM). If the phase legs are current controlled voltage sources, then the net power flowing into the individual phase legs over one cycle of the supply is zero for reactive power compensation applications under balanced conditions. Therefore the capacitor voltages for each of the H-bridges in the legs will stay at the correct value (ignoring losses). If the STATCOM is attempting to re-balance unbalanced supply currents it can be shown that average real power will flow into or out of individual H-bridge legs, resulting in capacitor constant build-up or decrease of the voltages on the leg capacitors. An example of this is shown in Figure 2 which shows the instantaneous power flowing into each of the legs of a set of wye connected current sources being

\footnotetext{
${ }^{1}$ One problem with the H-bridge converter is that the capacitors are subject to significant ripple current as compared to converters that have common energy storage for all the phases, such as the diode clamped or flying capacitor converter.
}

used to re-balance an open circuited phase. Notice that the average three phase power is zero (the top plot), but the average power of the plot denoted as power (CCCS_4p.CCCS_4p2) is positive, and that of power (CCCS_4p.CCCS_4p3) is negative, and power (cccs_4p.CCCS_4p4) is zero. Therefore two of the legs of the converter have average real power flow.

The use of a delta topology H-bridge STATCOM for unbalance compensation was published by Peng et al. [5]. Its control was based on passive delta compensator theory presented in [6] and originally attributed to Steinmetz. This theory allows one to design passive reactive circuits connected in delta to re-balance unbalanced currents. Because the compensator phase legs are composed of reactive components there is no average real power flowing into or out of individual phases. This concept was implemented in [5] by controlling the Hbridge based current controlled voltage sources to simulate the reactive elements designed using the approach in [6]. This implicitly means that on a per phase and three phase basis that there is no average power flowing into or out of the compensator. The structure of how such a compensator is connected to the grid and the general control structure proposed for the compensator is shown in Figure 3.

Remark 3: The use of the Miller/Steinmetz [6] technique in [5] implies that one must have an accurate model of the load impedances in order to calculate the required current references for each of the H-bridges. The control structure proposed in this paper detects the negative sequence currents on the load side of the converter and then uses these as the reference currents for the compensator. This idea in itself is not new, but can only be used for the H-bridge configuration in combination with the techniques developed in this paper to zero the average powers in each individual phase leg of the Hbridge converter. If this combined control is not applied then this technique cannot be used.

The remainder of this paper will develop two new strategies for ensuring that the average power in each phase leg of a H-bridge based multilevel converter STATCOM is zero for both the delta and wye configurations when used to rebalance an unsymmetrical supply. The control strategies use the concept of zero sequence injection to achieve zero average phase power. It is no surprise that the strategy for the delta connected compensator implements the same compensation 


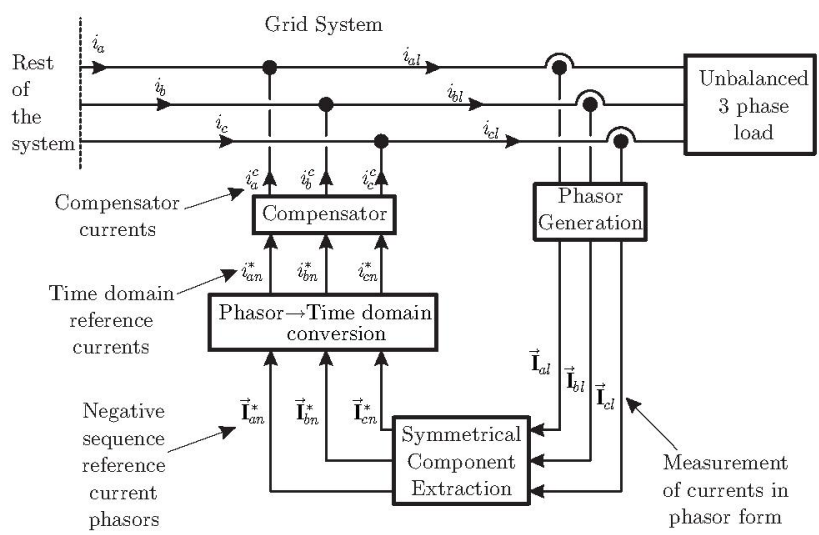

Fig. 3. Block diagram showing the connections of compensator to the grid system.

as the Peng[5] technique. However the implementation using zero sequence currents is more elegant and does not require identification of the load. The proposed control strategies can be combined with reactive power compensation algorithms and active filtering algorithms to implement a Universal STATCOM (U-STATCOM) using H-bridge multilevel converters.

\section{THE NEW AlgorithMS}

\section{A. Delta Connected H-Bridge STATCOM}

The control strategy employed in this paper is to extract the negative sequence currents present in the unbalanced load current using standard symmetrical component theory [7] and then inject these currents into the grid as $\overrightarrow{I_{\mathrm{a}}^{\mathrm{c}}}, \overrightarrow{I_{\mathrm{b}}^{\mathrm{c}}}$ and $\overrightarrow{I_{\mathrm{c}}^{\mathrm{c}}}$ using current controlled voltage sources implemented using H-bridge converters (these phasors are converted into time domain values to form the current source references). If the H-bridges are connected in a delta configuration then one of the current source values can be set to an arbitrary value and the other two are defined so that the external phase currents are equal to the desired negative sequence components. Alternatively, one can use a space vector approach to define the delta current source values as ${ }^{2}$ :

$$
\begin{aligned}
\vec{I}_{\mathrm{ab}} & =\frac{1}{3}\left(\overrightarrow{I_{\mathrm{a}}^{\mathrm{c}}}-\overrightarrow{I_{\mathrm{b}}^{\mathrm{c}}}\right) \\
\vec{I}_{\mathrm{bc}} & =\frac{1}{3}\left(\overrightarrow{I_{\mathrm{b}}^{\mathrm{c}}}-\overrightarrow{I_{\mathrm{c}}^{\mathrm{c}}}\right) \\
\vec{I}_{\mathrm{ca}} & =\frac{1}{3}\left(\overrightarrow{I_{\mathrm{c}}^{\mathrm{c}}}-\overrightarrow{I_{\mathrm{a}}^{\mathrm{c}}}\right)
\end{aligned}
$$

Remark 4: Whether using currents defined by (1)-(3), or setting an arbitrary current and defining the other two, a delta connected compensator one will end up with two or more of the current sources exchanging average real power with the grid. The total three phase average power is always zero. This is not a problem with inverter topologies with a common storage capacitor, but with H-bridges, as mentioned previously, this would mean that the capacitor voltages in individual legs

\footnotetext{
${ }^{2} \vec{I}$ denotes a phasor.
}

will be increasing or decreasing and clearly this situation is not sustainable. Therefore the situation is the same as that shown in Figure 2 for the wye connection.

We know from Peng's work [5] that it is possible to choose a combination of magnitude and phase for the currents in the current sources that satisfy the external condition that the negative sequence currents are supplied by the compensator and simultaneously that the individual average powers in the legs are zero.

We shall now introduce an extra degree of freedom into this situation by allowing a zero sequence current to flow around the delta. A zero sequence current will not affect the external phase current values (i.e the output negative sequence currents), and as we shall see can be used to get an average phase leg power of zero.

Let us now introduce some definitions - the desired compensator line currents are the negative sequence components and are defined $\mathrm{as}^{3}$ :

$$
\begin{aligned}
& \vec{I}_{\mathrm{an}}=\hat{I}_{\mathrm{n}} \angle \theta_{a n} \\
& \vec{I}_{\mathrm{bn}}=\hat{I}_{\mathrm{n}} \angle \theta_{b n}=\hat{I}_{\mathrm{n}} \angle\left(\theta_{a n}-\frac{4 \pi}{3}\right) \\
& \vec{I}_{\mathrm{cn}}=\hat{I}_{\mathrm{n}} \angle \theta_{c n}=\hat{I}_{\mathrm{n}} \angle\left(\theta_{a n}-\frac{2 \pi}{3}\right)
\end{aligned}
$$

The current references for the delta connected H-bridges can then be defined as per (1)-(3) with appropriate substitutions of (4)-(6) for $\overrightarrow{I_{\mathrm{a}}^{\mathrm{c}}}, \overrightarrow{I_{\mathrm{b}}^{\mathrm{c}}}$ and $\overrightarrow{I_{\mathrm{c}}^{\mathrm{c}}}$. An additional component to the $\mathrm{H}$-bridge reference values is a zero sequence current defined as:

$$
\vec{I}_{0}=\hat{I}_{0} \angle \alpha_{0}
$$

Including this the current reference for each of the current sources is now:

$$
\begin{aligned}
& \vec{I}_{\mathrm{ab} 0}=\vec{I}_{\mathrm{ab}}+\vec{I}_{0}=\frac{1}{3}\left(\vec{I}_{\mathrm{an}}-\vec{I}_{\mathrm{bn}}\right)+\vec{I}_{0} \\
& \vec{I}_{\mathrm{bc} 0}=\vec{I}_{\mathrm{bc}}+\vec{I}_{0}=\frac{1}{3}\left(\vec{I}_{\mathrm{bn}}-\vec{I}_{\mathrm{cn}}\right)+\vec{I}_{0} \\
& \vec{I}_{\mathrm{ca} 0}=\vec{I}_{\mathrm{ca}}+\vec{I}_{0}=\frac{1}{3}\left(\vec{I}_{\mathrm{cn}}-\vec{I}_{\mathrm{an}}\right)+\vec{I}_{0}
\end{aligned}
$$

Using (8)-(10) one can develop the expressions for the complex power in each phase of the delta:

$$
\begin{aligned}
& \vec{S}_{\mathrm{ab}}=\vec{V}_{\mathrm{ab}} \vec{I}_{\mathrm{ab} 0}^{*}=\vec{V}_{\mathrm{ab}}\left(\frac{1}{3}\left(\vec{I}_{\mathrm{an}}^{*}-\vec{I}_{\mathrm{bn}}^{*}\right)+\vec{I}_{0}^{*}\right) \\
& \vec{S}_{\mathrm{bc}}=\vec{V}_{\mathrm{bc}} \vec{I}_{\mathrm{bc} 0}^{*}=\vec{V}_{\mathrm{bc}}\left(\frac{1}{3}\left(\vec{I}_{\mathrm{bn}}^{*}-\vec{I}_{\mathrm{cn}}^{*}\right)+\vec{I}_{0}^{*}\right) \\
& \vec{S}_{\mathrm{ca}}=\vec{V}_{\mathrm{ca}} \vec{I}_{\mathrm{ca} 0}^{*}=\vec{V}_{\mathrm{bc}}\left(\frac{1}{3}\left(\vec{I}_{\mathrm{cn}}^{*}-\vec{I}_{\mathrm{an}}^{*}\right)+\vec{I}_{0}^{*}\right)
\end{aligned}
$$

where $\vec{V}_{\mathrm{ab}}, \vec{V}_{\mathrm{bc}}$, and $\vec{V}_{\mathrm{ca}}$ are defined as follows:

$$
\begin{aligned}
& \vec{V}_{\mathrm{ab}}=\hat{V}_{\mathrm{ab}} \angle \theta_{a b} \\
& \vec{V}_{\mathrm{bc}}=\hat{V}_{\mathrm{bc}} \angle\left(\theta_{a b}-\frac{2 \pi}{3}\right) \\
& \vec{V}_{\mathrm{ca}}=\hat{V}_{\mathrm{ca}} \angle\left(\theta_{a b}-\frac{4 \pi}{3}\right)
\end{aligned}
$$

${ }^{3} \hat{I}$ denotes the rms magnitude of a phasor. 
Because of the symmetry in the expressions we shall only consider the 'ab' expression. Expanding the expression for $\vec{S}_{\mathrm{ab}}$ we can write:

$$
\begin{aligned}
\vec{S}_{\mathrm{ab}}=\frac{\vec{V}_{\mathrm{ab}}}{3}\left(\hat{I}_{\mathrm{an}} \angle\left(\theta_{a b}-\theta_{a n}\right)-\hat{I}_{\mathrm{bn}} \angle\left(\theta_{a b}-\theta_{b n}\right)\right. \\
\left.+3 \hat{I}_{0} \angle\left(\theta_{a b}-\alpha_{0}\right)\right)
\end{aligned}
$$

We are specifically interested in the real power in the current source for each leg - we want the average real power to be zero. Therefore we want the expression:

$$
P_{a b}=\Re\left\{\vec{S}_{\mathrm{ab}}\right\}
$$

Recognising from (5) and (6) that:

$$
\begin{aligned}
& \theta_{b n}=\theta_{a n}+\frac{2 \pi}{3}=\theta_{a n}-\frac{4 \pi}{3} \\
& \theta_{c n}=\theta_{a n}+\frac{4 \pi}{3}=\theta_{a n}-\frac{2 \pi}{3}
\end{aligned}
$$

we can write:

$$
\begin{aligned}
P_{a b}=\frac{\hat{V}_{\mathrm{ab}} \hat{I}_{\mathrm{n}}}{3}\left[\cos \left(\theta_{a b}-\theta_{a n}\right)\right. & -\cos \left(\theta_{a b}-\theta_{a n}-\frac{2 \pi}{3}\right) \\
& \left.+\frac{3 \hat{I}_{0}}{\hat{I}_{\mathrm{n}}} \cos \left(\theta_{a b}-\alpha_{0}\right)\right]
\end{aligned}
$$

Considering the term $\cos \left(\theta_{a b}-\theta_{a n}\right)-\cos \left(\theta_{a b}-\theta_{a n}-\frac{2 \pi}{3}\right)$ from (21), and using the trigonometric relation:

$$
\cos v-\cos u=2 \sin \left(\frac{u+v}{2}\right) \sin \left(\frac{u-v}{2}\right)
$$

we can write that:

$$
\begin{aligned}
\cos \left(\theta_{a b}-\theta_{a n}\right)-\cos \left(\theta_{a b}\right. & \left.-\theta_{a n}-\frac{2 \pi}{3}\right) \\
& =-\sqrt{3} \sin \left(\theta_{a b}-\theta_{a n}-\frac{\pi}{3}\right)
\end{aligned}
$$

This can be further manipulated using $\sin x=\cos \left(x-\frac{\pi}{2}\right)$ to give the expression in a cosine form:

$$
\begin{aligned}
\cos \left(\theta_{a b}-\theta_{a n}\right)-\cos \left(\theta_{a b}-\theta_{a n}-\frac{2 \pi}{3}\right) & \\
= & -\sqrt{3} \cos \left(\theta_{a b}-\theta_{a n}-\frac{5 \pi}{6}\right)
\end{aligned}
$$

Therefore (21) can be written as:

$$
\begin{aligned}
P_{a b}=\frac{\hat{V}_{\mathrm{ab}} \hat{I}_{\mathrm{n}}}{3}\left(\frac{3 \hat{I}_{0}}{\hat{I}_{\mathrm{n}}} \cos \left(\theta_{a b}-\alpha_{0}\right)\right. & \\
& \left.\quad-\sqrt{3} \cos \left(\theta_{a b}-\theta_{a n}-\frac{5 \pi}{6}\right)\right)
\end{aligned}
$$

Summary 1: The real powers for each of the phases can therefore be written as:

$$
\begin{aligned}
P_{a b}=\hat{V}_{\mathrm{ab}} \hat{I}_{\mathrm{n}}\left(\hat{I}_{0}^{\prime} \cos \left(\theta_{a b}-\alpha_{0}\right)\right. & \\
& \left.-\frac{1}{\sqrt{3}} \cos \left(\theta_{a b}-\theta_{a n}-\frac{5 \pi}{6}\right)\right)
\end{aligned}
$$

$$
\begin{aligned}
P_{b c}=\hat{V}_{\mathrm{bc}} \hat{I}_{\mathrm{n}}\left(\hat { I } _ { 0 } ^ { \prime } \operatorname { c o s } \left(\theta_{a b}\right.\right. & \left.+\frac{4 \pi}{3}-\alpha_{0}\right) \\
& \left.-\frac{1}{\sqrt{3}} \cos \left(\theta_{a b}-\theta_{a n}-\frac{\pi}{6}\right)\right) \\
P_{c a}=\hat{V}_{\mathrm{ca}} \hat{I}_{\mathrm{n}}\left(\hat { I } _ { 0 } ^ { \prime } \operatorname { c o s } \left(\theta_{a b}\right.\right. & \left.+\frac{2 \pi}{3}-\alpha_{0}\right) \\
& \left.-\frac{1}{\sqrt{3}} \cos \left(\theta_{a b}-\theta_{a n}-\frac{\pi}{2}\right)\right)
\end{aligned}
$$

where the normalised zero sequence current is $\hat{I}_{0}^{\prime}=\frac{\hat{I}_{0}}{\hat{I}_{\mathrm{n}}}$

Remark 5: We know from [5] and [6] that it is possible to have zero average power in each phase. Therefore the objective is to find the $\vec{I}_{0}$ to achieve $P_{a b}=P_{b c}=P_{c a}=0$. This means that:

$$
\begin{array}{r}
\hat{I}_{0}^{\prime} \cos \gamma-\frac{1}{\sqrt{3}} \cos \left(\beta-\frac{5 \pi}{6}\right)=0 \\
\hat{I}_{0}^{\prime} \cos \left(\gamma+\frac{4 \pi}{3}\right)-\frac{1}{\sqrt{3}} \cos \left(\beta-\frac{\pi}{6}\right)=0 \\
\hat{I}_{0}^{\prime} \cos \left(\gamma+\frac{2 \pi}{3}\right)-\frac{1}{\sqrt{3}} \cos \left(\beta+\frac{\pi}{2}\right)=0
\end{array}
$$

where $\beta=\theta_{a b}-\theta_{a n}$ and $\gamma=\theta_{a b}-\alpha_{0}$. (31):

We can make the following definitions for parts of (29)-

$$
\begin{aligned}
& x=\frac{1}{\sqrt{3}} \cos \left(\beta-\frac{5 \pi}{6}\right) \\
& y=\frac{1}{\sqrt{3}} \cos \left(\beta-\frac{\pi}{6}\right) \\
& z=\frac{1}{\sqrt{3}} \cos \left(\beta+\frac{\pi}{2}\right)
\end{aligned}
$$

Therefore (29)-(31) can be rewritten as:

$$
\begin{array}{r}
\hat{I}_{0}^{\prime} \cos \gamma-x=0 \\
\hat{I}_{0}^{\prime} \cos \left(\gamma+\frac{4 \pi}{3}\right)-y=0 \\
\hat{I}_{0}^{\prime} \cos \left(\gamma+\frac{2 \pi}{3}\right)-z=0
\end{array}
$$

Because $x+y+z=0$ we can write (35) as:

$$
\cos \gamma=-\frac{y+z}{\hat{I}_{0}^{\prime}}
$$

Similarly, manipulating (36) (or (37)) as follows:

$$
\begin{array}{r}
\hat{I}_{0}^{\prime}\left(\cos \gamma \cos \left(\frac{4 \pi}{3}\right)-\sin \gamma \sin \left(\frac{4 \pi}{3}\right)\right)-y=0 \\
\therefore \hat{I}_{0}^{\prime}\left(-\frac{1}{2} \cos \gamma+\frac{\sqrt{3}}{2} \sin \gamma\right)=0 \\
\therefore \hat{I}_{0}^{\prime}\left(\frac{1}{2}\left(\frac{y+z}{\hat{I}_{0}^{\prime}}\right)+\frac{\sqrt{3}}{2} \sin \gamma\right)-y=0
\end{array}
$$

Equation (41) can be rearranged as:

$$
\sin \gamma=\frac{1}{\sqrt{3} \hat{I}_{0}^{\prime}}(y-z)
$$


Remark 6: Equations (38) and (42) constitute two equations with two unknowns (i.e. $\hat{I}_{0}^{\prime}$ and $\alpha_{0}$ ), which can be solved to give an average real phase power of zero.

From (38) and (42) one can write:

$$
\hat{I}_{0}^{\prime}=-\frac{y+z}{\cos \gamma}
$$

and substituting into (42) gives:

$$
\tan \gamma=-\frac{1}{\sqrt{3}} \frac{y-z}{y+z}
$$

Summary 2: Equations (43) and (44) are the two equations that give $\hat{I}_{0}$ and $\alpha_{0}$ since $\hat{I}_{0}=\hat{I}_{0}^{\prime} \hat{I}_{\mathrm{n}}$, and $\alpha_{0}=\theta_{a b}-\gamma$, where $\theta_{a b}$ is known.

\section{B. Wye Connected H-bridge STATCOM}

In the case of the wye connected H-bridge STATCOM the currents in each of the phase legs is simply the reference negative sequence currents that have to be injected into the network - i.e. the currents in equations (4)-(6). Let us define a set of positive sequence voltages relative to an arbitrary reference angle:

$$
\begin{aligned}
& \vec{V}_{\mathrm{a}}=\hat{V} \angle \theta_{a} \\
& \vec{V}_{\mathrm{b}}=\hat{V} \angle\left(\theta_{a}-\frac{2 \pi}{3}\right) \\
& \left.\vec{V}_{\mathrm{c}}=\hat{V} \angle \theta_{a}-\frac{4 \pi}{3}\right)
\end{aligned}
$$

We can define a zero sequence phase voltage as:

$$
\vec{V}_{0}=\hat{V}_{0} \angle \alpha_{0}
$$

Therefore the actual resultant phase voltages are defined as:

$$
\begin{aligned}
& \vec{V}_{\mathrm{a}}^{\prime}=\vec{V}_{\mathrm{a}}+\vec{V}_{0} \\
& \vec{V}_{\mathrm{b}}^{\prime}=\vec{V}_{\mathrm{b}}+\vec{V}_{0} \\
& \vec{V}_{\mathrm{c}}^{\prime}=\vec{V}_{\mathrm{b}}+\vec{V}_{0}
\end{aligned}
$$

With these definitions, we can now calculate the powers in each of the phases of the compensator, assuming that each phase leg is is conducting the relevant negative sequence current:

$$
\begin{aligned}
& \vec{S}_{\mathrm{a}}=\vec{V}_{\mathrm{a}}^{\prime} \vec{I}_{\mathrm{an}}^{*} \\
& \vec{S}_{\mathrm{b}}=\vec{V}_{\mathrm{b}}^{\prime} \vec{I}_{\mathrm{bn}}^{*} \\
& \vec{S}_{\mathrm{c}}=\vec{V}_{\mathrm{c}}^{\prime} \vec{I}_{\mathrm{cn}}^{*}
\end{aligned}
$$

Taking the real component of $\vec{S}_{\mathrm{a}}, \vec{S}_{\mathrm{b}}$ and $\vec{S}_{\mathrm{c}}$ we can write:

$$
\begin{array}{rr}
\Re\left\{\vec{S}_{\mathrm{a}}\right\}=\hat{V} \hat{I}_{\mathrm{n}}\left[\cos \left(\theta_{a n}-\theta_{a}\right)\right. & \\
& \left.+\frac{\hat{V}_{0}}{\hat{V}} \cos \left(\theta_{a n}-\alpha_{0}\right)\right]
\end{array}
$$

$$
\begin{array}{r}
\Re\left\{\vec{S}_{\mathrm{b}}\right\}=\hat{V} \hat{I}_{\mathrm{n}}\left[\cos \left(\theta_{a n}-\theta_{a}-\frac{2 \pi}{3}\right)\right. \\
\left.+\frac{\hat{V}_{0}}{\hat{V}} \cos \left(\theta_{a n}-\alpha_{0}-\frac{4 \pi}{3}\right)\right] \\
\Re\left\{\vec{S}_{\mathrm{c}}\right\}=\hat{V} \hat{I}_{\mathrm{n}}\left[\cos \left(\theta_{a n}-\theta_{a}+\frac{2 \pi}{3}\right)\right. \\
\left.+\frac{\hat{V}_{0}}{\hat{V}} \cos \left(\theta_{a n}-\alpha_{0}+\frac{4 \pi}{3}\right)\right]
\end{array}
$$

Taking (55) and (56) and equating to zero (so that we have two equations in two unknowns $-\hat{V}_{0}$ and $\alpha_{0}$ ) we can write:

$$
\begin{aligned}
\cos \left(\theta_{a n}-\theta_{a}\right) & =-\frac{\hat{V}_{0}}{\hat{V}} \cos \left(\theta_{a n}-\alpha_{0}\right) \\
\cos \left(\theta_{a n}-\theta_{a}-\frac{2 \pi}{3}\right)= & -\frac{\hat{V}_{0}}{\hat{V}} \cos \left(\theta_{a n}-\alpha_{0}-\frac{4 \pi}{3}\right) \\
\therefore \cos \left(\theta_{a n}-\theta_{a}-\frac{2 \pi}{3}\right)= & \frac{\hat{V}_{0}}{2 \hat{V}} \cos \left(\theta_{a n}-\alpha_{0}\right) \\
& +\frac{\sqrt{3} \hat{V}_{0}}{2 \hat{V}} \sin \left(\theta_{a n}-\alpha_{0}\right)
\end{aligned}
$$

Substituting (58) into (60) we can write after some simplification:

$$
\sin \left(\theta_{a n}-\theta_{a}\right)=\frac{\hat{V}_{0}}{\hat{V}} \sin \left(\theta_{a n}-\alpha_{0}\right)
$$

Rearranging (58) to make $\hat{V}_{0} / \hat{V}$ the subject of the expression, and then substituting into (61) and rearranging we can write:

$$
\tan \left(\theta_{a n}-\alpha_{0}\right)=-\tan \left(\theta_{a n}-\theta_{a}\right)
$$

Taking the inverse tan of both sides of (62) we can obviously write:

$$
\begin{aligned}
\theta_{a n}-\alpha_{0} & =-\left(\theta_{a n}-\theta_{a}\right) \\
\therefore \alpha_{0} & =2 \theta_{a n}-\theta_{a}=\theta_{a n}+\zeta
\end{aligned}
$$

where $\zeta=\theta_{a n}-\theta_{a}$, the angle between the $\vec{V}_{\mathrm{a}}$ phasor (i.e. the balanced system phasor) and the negative sequence current phasor $\vec{I}_{\text {an }}$.

Remark 7: Note that $\theta_{a n}-\theta_{a}=\zeta$ is an angle that is fixed by the relationship between the negative sequence current phasors and the positive sequence voltage phasor - it is established by external circuit conditions.

Substituting (64) into (58) we can write:

$$
\begin{aligned}
\cos \left(\theta_{a n}-\theta_{a}\right) & =-\frac{\hat{V}_{0}}{\hat{V}} \cos \left(\theta_{a n}-\theta_{a n}-\zeta\right) \\
\therefore \cos \zeta & =-\frac{\hat{V}_{0}}{\hat{V}} \cos \zeta \\
\text { or } \hat{V}_{0} & =-\hat{V} \\
\therefore \vec{V}_{0} & =-\hat{V} \angle \alpha_{0}=\hat{V} \angle\left(\alpha_{0}+\pi\right)
\end{aligned}
$$




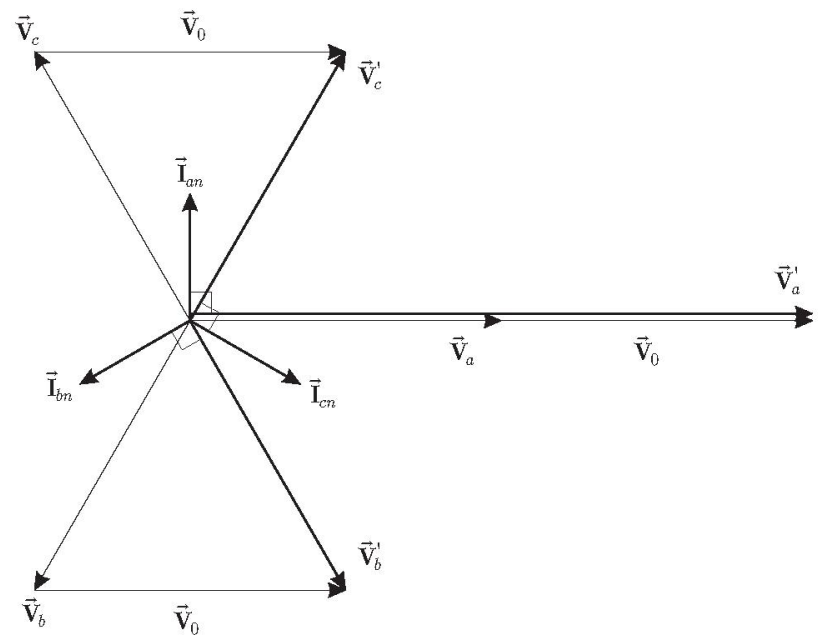

Fig. 4. Phasor diagram of balancing in a wye connected STATCOM with zero sequence voltage injection.

Remark 8: Equations (64) and (67) say that the magnitude of the zero sequence is always equal to the magnitude of the balanced phasor voltages, and the angle of the phasor is always $\zeta=\theta_{a n}-\theta_{a}$ from the $\vec{V}_{\text {a }}$ phasor.

Summary 3: For the wye connected H-bridges the following conditions have to apply for the injected zero sequence voltage to obtain zero average power in each of the H-bridges:

$$
\begin{gathered}
\hat{V}_{0}=-\hat{V} \\
\alpha_{0}=\theta_{a n}+\zeta
\end{gathered}
$$

where $\zeta=\theta_{a n}-\theta_{a}$ (the angle between the negative sequence current to be injected and the positive sequence voltage phasor of the grid phase voltage), and $\hat{V}$ is the magnitude of the positive sequence phase voltage. The $\theta_{a}$ angle is the angle of the the positive sequence ' $a$ ' phase voltage with respect to an arbitrary reference.

Example 1: Consider the situation of $\theta_{a}=0$ and $\zeta=$ $\pi / 2 \Rightarrow \theta_{\text {an }}=\pi / 2$, then $\vec{V}_{0}=-\hat{V} \angle \alpha_{0}=-\hat{V} \angle \pi=\hat{V} \angle 0$ is in phase with $\vec{V}_{\text {a }}$. Therefore the resultant voltage across phase 'a' is $\vec{V}_{\mathrm{a}}^{\prime}=\vec{V}_{\mathrm{a}}+\vec{V}_{\mathrm{a}}$ - twice the normal phase voltage value. This effectively means that the star point of the wye compensator has moved. The other two phases have the normal voltage magnitude across them. A phasor diagram of this situation is shown in Figure 4.

Note that the negative sequence currents are orthogonal to the respective phase voltage, therefore there is no average real power flowing to the phase legs of the compensator. This can be shown to work for any arbitrary unbalanced load.

\section{Duality, Ratings and Implementation Issues}

There are some interesting comparisons that can be made between the ratings for the H-bridges for the delta and wye configurations:

- The delta converter configuration has to have a $\sqrt{3}$ higher voltage rating compared to the wye connected

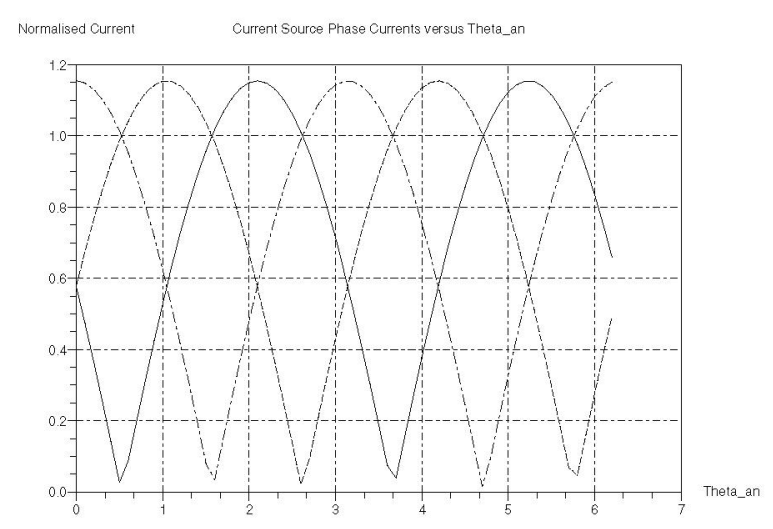

Fig. 5. Normalised delta current source current amplitudes with zero sequence current injection versus negative sequence phase angle.

converter operating on a balanced system - this is the standard difference between delta and wye ratings. The zero sequence current injected around the delta results in a current rating for each leg that is 1.15 times the negative sequence current amplitude from the converter, which is $15 \%$ larger than the current rating for the wye connected converter.

- The wye connection with zero sequence voltage has to have all of the legs of the converter rated at twice the normal phase voltage rating, which is 1.15 times the lineto-line rating of the delta converter (i.e. 15\% larger than the delta rating). The current rating is the same as the required negative sequence current amplitude.

Figure 5 illustrates the above mentioned rating for the delta converter by showing how the normalised currents in each of the current sources varies with the phase of the negative sequence current when there is zero sequence injection. Note that ' 1 ' on this corresponds to the amplitude of the negative sequence current to be supplied by the converter. One can see that the maximum value of the current is 1.15 - i.e. $15 \%$ larger than the negative sequence current to be supplied.

The two situations are duals of each other - the delta converter needs the H-bridge legs to be current rated at 1.15 times that of the wye connection, and the wye connection requires that H-bridge legs to be voltage rated at 1.15 times the delta connected bridges.

Remark 9: Contrary to what one may have first thought, the use of the wye connection does not improve the situation with respect to the voltage rating of the converter, due to the injection of the zero sequence voltage. The economic implications of the different ratings with respect to current and voltage is not clear though. For example, the high voltage rating of the wye connected converter could result in more $\mathrm{H}$ bridges in each leg, or alternatively a higher voltage rating for fewer bridges. This clearly has an economic cost.

For the delta converter, the current rating of each bridge is much be higher and one would normally think that this 
would be lower cost than having the higher voltage rating for the wye connection. However, higher current rating not only affects the rating of the semiconductors in the bridges, but more importantly affects the current ripple rating of the capacitors in each bridge. H-bridge multilevel architectures are particularly demanding on the capacitors because of the high ripple currents inherent for the topology. Therefore a higher current rating will have significant implications on the cost of the capacitors.

For either converter type there are significant economic costs to achieve re-balancing.

In order to implement the delta connection, the normal negative sequence reference currents are augmented with the desired zero sequence current calculated using equations (43) and (44). In the case of the wye connection the situation is a little different. In this case two of the converter legs are implemented using two currents sources and the remaining leg is a voltage source. The presence of the voltage source for one of the phase legs allows the injection of the zero sequence voltage, whilst the two current sources allow the desired line current outputs to be achieved. The presence of the voltage source together with the floating star point means that Kirchoff's Current Law is automatically satisfied at this point, and the current through the voltage source is the desired phase current.

\section{Simulation Studies}

In order to test the algorithms a number of simulations were carried out using the Saber ${ }^{\circledR}$ simulation package. The simulation also simulated the phasor sensing system using a Karimi-Ghartemani Phase Locked Loop [8]. Different unbalance situations were considered.

\section{A. Delta Connection}

Similar to the case in Peng[5], the open circuit phase case was considered as an extreme example for the delta case. Figures 6 and 7 show Saber ${ }^{\circledR}$ simulation results for this situation. Figure 6 shows the source side phase currents. Notice that up to $200 \mathrm{msec}$ there is no current flowing in phase ' $\mathrm{a}$ ', and after $200 \mathrm{msec}$, when the compensator is turned on, the currents are balanced. At $500 \mathrm{msec}$ the zero sequence current is injected into the delta and as expected the external compensator currents are unaffected. Figure 7 shows the powers in the individual phase current sources under these conditions. One of the current sources has no average power through it, whilst the other two have average positive and negative powers up to $500 \mathrm{msec}$. After the zero sequence current is injected all the current sources have an average power of zero. ${ }^{4}$

Remark 10: The results in Figures 6 and 7 are similar to those in [5]. The currents in the individual current sources with the zero sequence injection are exactly the same as those in [5]. However the control technique is a pure feedback approach, and does not require load impedance identification.

\footnotetext{
${ }^{4}$ Note from Figure 7 that the oscillation of the powers is not equal in each of the current sources.
}

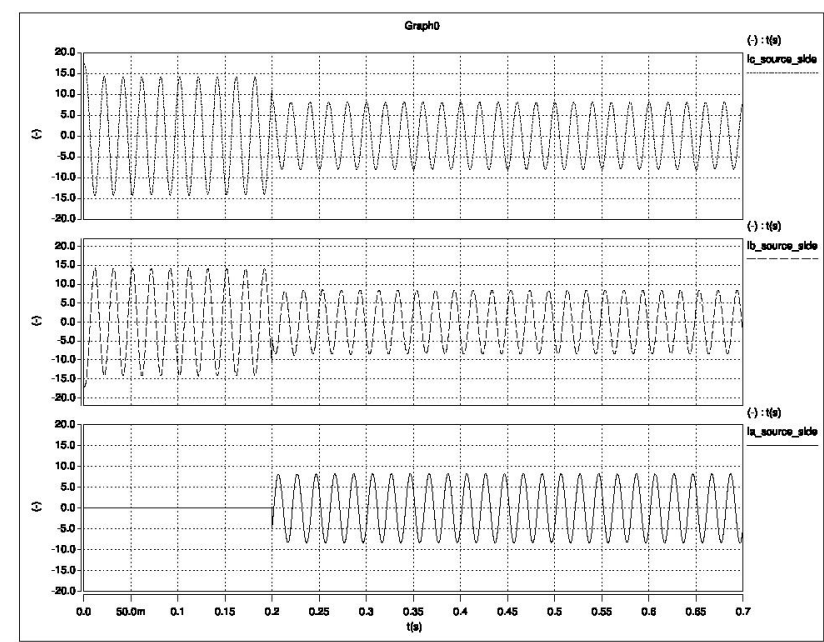

Fig. 6. Source side line currents for delta connected compensator. Compensator turned on at $200 \mathrm{msec}$. Zero sequence injection at $500 \mathrm{msec}$.

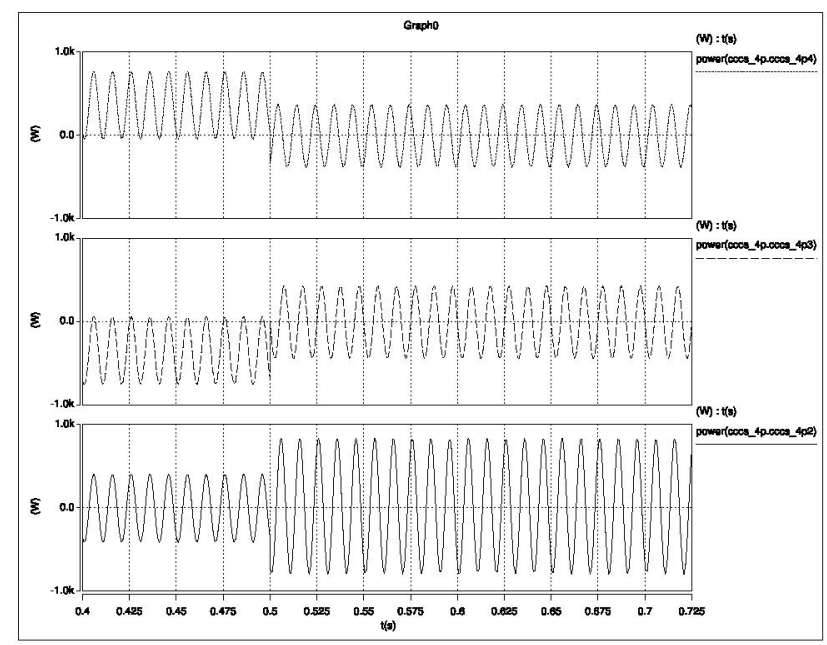

Fig. 7. Delta compensator current source powers. Zero sequence injection at $500 \mathrm{msec}$.

Figure 8 shows the effect of the zero sequence current injection on the currents in the current sources in this particular case. At 0.2 seconds the currents are simply those required to produce the desired negative sequence output currents without power balancing. At 0.5 secs one can see the increase in some currents and decrease in others when the zero sequence is injected. There is also a phase shift in the currents. In this particular case the amplitude of the zero sequence current was approximately 4.7 Amps.

\section{B. Wye Connection}

A simulation of a wye connected compensator operating with zero sequence voltage injection and an unbalanced load of the form $Z_{a}=10+j \omega(0.001), Z_{b}=15+j \omega(0.1)$ and $Z_{c}=5+j \omega(0.0001)$ can be seen in Figures 9 and 10. These figures show the voltages across the controlled current sources of the compensator, the star point voltage relative to the normal balanced star point and the powers of each current source. 


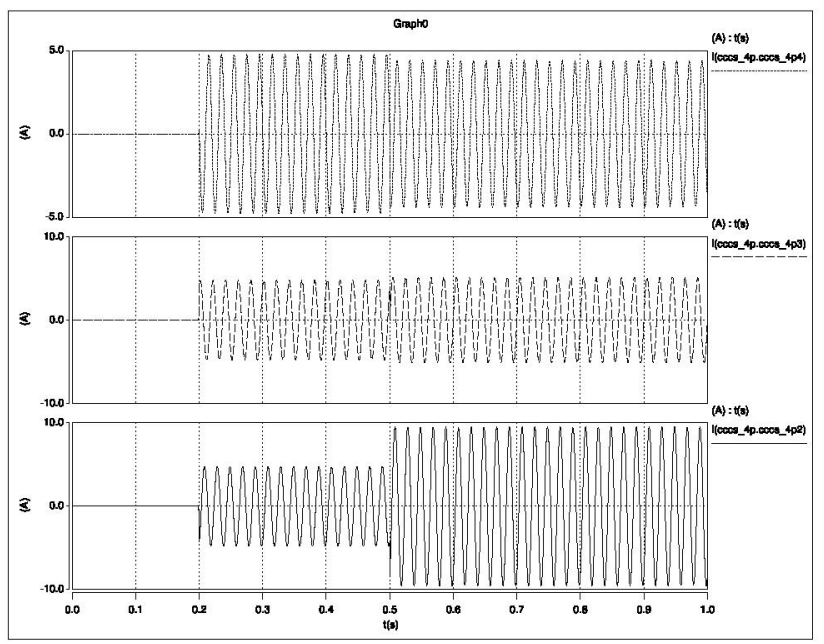

Fig. 8. Currents in the current sources with a delta compensator, with and without zero sequence current injection.

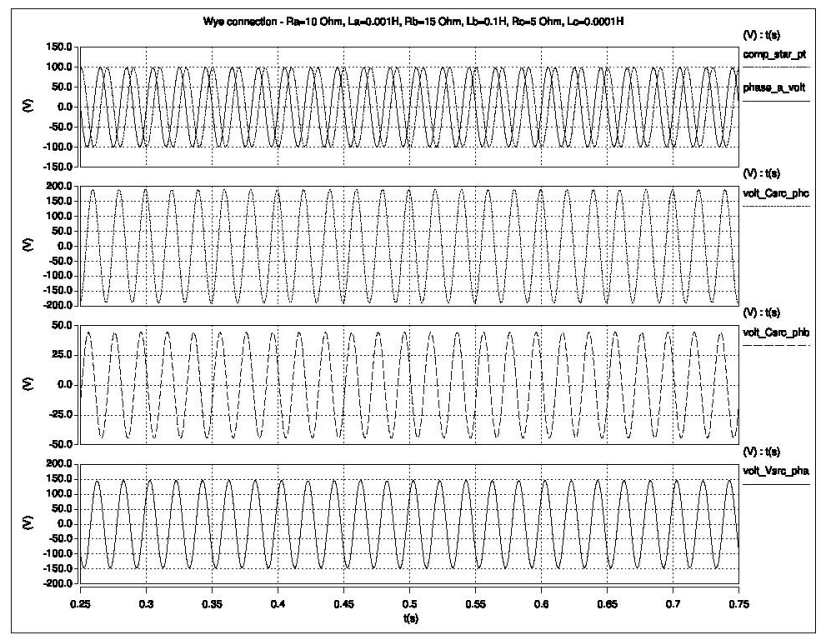

Fig. 9. Voltages for a wye connected compensator with zero sequence injection and a general unbalanced load.

Although the plots are not shown, the wye converter, similar to the delta case, balances the unbalanced currents present in this situation.

Remark 11: Notice from Figure 9 that the zero sequence voltage (essentially the voltage of the star point) has the same magnitude as the normal phase ' $a$ ' voltage. The phase voltages actually appearing across the current sources are very different with a peak amplitude difference of 100 Volts. Figure 10 shows that the zero sequence voltage injection has resulted in the average powers in the three current sources being zero.

\section{CONClusions AND CONTRIBUtions}

The main contributions of this paper are:

- The development of a new control concept based on the injection of zero sequence currents and voltages to give an average zero real power in the phase legs of delta and wye connected H-bridge multilevel converters when used to re-balance unbalanced supply currents.

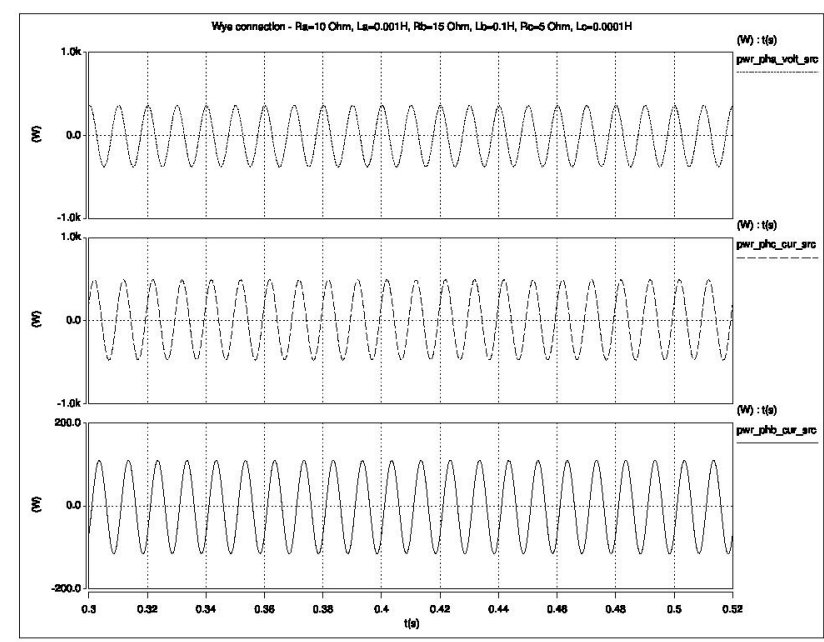

Fig. 10. Powers for a wye connected compensator with zero sequence voltage injection and a general unbalanced load.

- Proof that for both the delta and wye connected converter that the zero sequence injection gives zero average power in each individual phase leg of the H-bridge converter.

- Simulation of the control strategies developed using a control topology based on using the negative sequence load currents as the references.

- The duality on the ratings of the delta and wye converters is explained.

In summary, this paper has shown that it is possible to use the H-bridge multilevel topology, connected in either delta or wye, to balance unbalanced currents in a supply network using a feedback control structure and zero seqence injection.

\section{REFERENCES}

[1] C. Yung, "Stopping a costly leak: The effects of unbalanced voltage on the life and efficiency of three-phase electric motors." US Department of Energy website: http://www.oit.doe.gov/bestpractices/energymatters/wint2005_motors.shmtl, Winter 2005.

[2] "Single phasing and phase unbalance" Internet: http://www.wisconsinpublicservice.com/business/phase.asp, 2005.

[3] A. von Jouanne and B. Banerjee, "Assessment of voltage unbalance," IEEE Transactions on Power Delivery, vol. 16, no. 4, pp. 782-790, 2001.

[4] J. Rodriguez, J. S. Lai, and F. Z. Peng, "Multilevel inverters: A survey of topologies, controls, and applications," IEEE Transactions on Industrial Electronics, vol. 49, pp. 724-738, August 2002.

[5] F. Peng and J. Wang, "A universal statcom with delta-connected cascade multilevel inverter," in 35th Annual IEEE Power Electronics Specialist Conference, Aachen, Germany, pp. 3529-3533, IEEE, 2004.

[6] T. J. E. Miller, Reactive Power Control in Electric Systems. John Wiley and Sons, 1982. ISBN: 0-471-86933-3.

[7] W. D. Stevenson, Elements of Power System Analysis. McGraw-Hill, 1975. ISBN 0-07-061285-4.

[8] M. Karimi-Ghartmani, M. Iravani, and F. Katiraei, "Extraction of signals for harmonics, reactive current and network-unbalance compensation," IEE Proceedings - Generation, Transmission and Distribution, vol. 152, pp. 137-143, January 2005. 